$05.1 ; 15.2$

\title{
Тестирование на изгиб наноразмерных консолей в атомно-силовом микроскопе
}

\author{
(C) А.В. Анкудинов ${ }^{1}$, М.М. Халисов ${ }^{1,2}$ \\ ${ }^{1}$ Физико-технический институт им. А.Ф. Иоффер РАН, Санкт-Петербург, Россия \\ ${ }^{2}$ Институт фризиологии им. И.П. Павлова РАН, Санкт-Петербург, Россия \\ E-mail: alexander.ankudinov@mail.ioffe.ru
}

Поступило в Редакцию 1 сентября 2021 г.

В окончательной редакции 18 октября 2021 г.

Принято к публикации 20 октября 2021 г.

\begin{abstract}
Консоли и мостики из наносвитков $\mathrm{MgNi}_{2} \mathrm{Si}_{2} \mathrm{O}_{5}(\mathrm{OH})_{4}$ испытывались в атомно-силовом микроскопе на изгиб. Условия закрепления объектов анализировались по данным испытаний и учитывались в расчете модуля Юнга наносвитков. Результаты для консолей и мостиков хорошо согласовывались, если вторые моделировались как трехпролетные балки, а первые - как балки на упругом основании с выносной консолью.
\end{abstract}

Ключевые слова: атомно-силовая микроскопия, изгиб, наносвиток, модуль Юнга, функции Крылова.

DOI: 10.21883/PJTF.2022.03.51978.19010

Модуль Юнга подвешенного квазиодномерного нанообъекта (трубки, стержня, свитка) можно определить с помощью атомно-силовой микроскопии (АСМ) по методике испытаний на изгиб [1]. Консоли и мостики формируются в результате высыхания коллоидной капли тестируемых объектов на различных подложках с углублениями [2,3]. Методика опирается на АCMизмерения профиля жесткости объекта и теорию слабых изгибов стержней [4]. Основную погрешность измерений вызывают неизвестные условия закрепления. Расчеты модуля Юнга мостика, если считать его опертой или защемленной балкой, различаются в 4 раза. Такая неопределенность устраняется $[5,6]$ путем сравнения измеренного профиля с профилем жесткости центрального пролета модельной трехпролетной балки (рис. 1, $a$, вверху). Если удлинять боковые пролеты, центральный пролет плавно перейдет из защемленного состояния в опертое. Значение $\lambda=L / l$, согласующее теорию и измерение, применяется для корректировки модуля Юнга. На практике могут деформироваться как подвешенный объект, так и подложка. В этом случае полезна модель, когда объект оперт на упругое основание (рис. 1, $a$, внизу). В этой модели, однако, нет компактной формулы для профиля жесткости [7]. Посредством АСМ на изгиб испытывались не только мостики, но и консоли [3]. На результат измерений также влияют условия закрепления консолей на подложке. Устранить связанную с этим неопределенность значений модуля Юнга консоли было целью настоящей работы.

Вверху на рис. $1, b$ показана схема подпертой консольной балки (модель I): первый пролет защемлен в точке $x=-L$, оперт в точке $x=0 ;$ второй пролет, консоль длиной $l$, оперт в точке $x=0$, сила $F$ приложена в точке $x=X, X \in(0, l]$; модуль Юнга $E$ и момент инерции балки $I$. Формулу изгиба консоли $z(x)$ в этой статически неопределимой задаче можно получить, применяя метод сложения действия сил [8] (вывод формулы представлен в дополнительных материалах в онлайн-версии статьи). Приведем ее

$$
z(x)=F \frac{3 X L x+6 X x^{2}-2 x^{3}}{12 E I}, \quad x \in[0, X], X \in(0, l] .
$$

Прямо зависимость (1) в АСМ не проверить, но можно измерить жесткость или обратно пропорциональный ей изгиб (деформацию) консоли в точке нагрузки $x=X$ :

$$
z(X)=F \frac{3 L X^{2}+4 X^{3}}{12 E I} .
$$

Нормируя $z(X)$ на максимум $z(l)$, а $X$ - на длину консоли $l$, получаем для модели I формулу подгонки
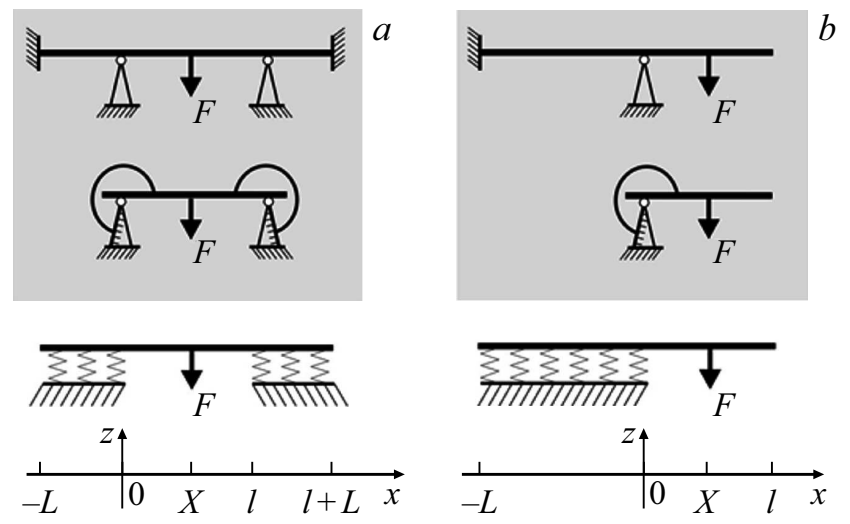

Pис. 1. $a-$ условия закрепления наномостика: схемы трехпролетной балки (вверху) и балки на упругом основании (внизу) рассматривались в [5] и [7] (схемы вверху и в центре балка на кольцевых пружинах, соответствующих граничным условиям $z^{\mathrm{II}}(0)=4 z^{\mathrm{I}}(0) / L$ и $z^{\mathrm{II}}(l)=-4 z^{\mathrm{I}}(l) / L,-$ эквивалентны). $b$ - условия закрепления консоли (настоящая работа): модель I - схемы вверху и в центре, модель II - схема внизу. 
Функции Крылова

\begin{tabular}{r|c|c}
\hline$i$ & $Y_{i}$ & $K_{i}$ \\
\hline 1 & $Y_{1}(x)=\cosh x \cos x$ & $K_{1}(x)=\frac{1}{2}(\cosh x+\cos x)$ \\
2 & $Y_{2}(x)=\frac{1}{2}(\cosh x \sin x+\sinh x \cos x)$ & $K_{2}(x)=\frac{1}{2}(\sinh x+\sin x)$ \\
3 & $Y_{3}(x)=\frac{1}{2} \sinh x \sin x$ & $K_{3}(x)=\frac{1}{2}(\cosh x-\cos x)$ \\
4 & $Y_{4}(x)=\frac{1}{4}(\cosh x \sin x-\sinh x \cos x)$ & $K_{4}(x)=\frac{1}{2}(\sinh x-\sin x)$
\end{tabular}

профиля изгиба к эксперименту

$$
\xi_{\mathrm{I}}(\chi)=\frac{4}{4+3 \lambda} \chi^{3}+\frac{3 \lambda}{4+3 \lambda} \chi^{2}, \quad \chi=\frac{X}{l}, \quad \lambda=\frac{L}{l},
$$

и, подставляя в (2) $X=l$, выражения для расчета модуля Юнга $E$

$$
\begin{gathered}
E=\frac{F}{z(l)} \frac{l^{3}}{3 I} \frac{4+3 \lambda}{4}=E_{0} \Phi_{\mathrm{I}}, \\
\Phi_{\mathrm{I}}=\frac{4+3 \lambda}{4}, \quad E_{0}=\frac{F}{D^{\max }} \frac{64 l^{3}}{3 \pi d^{4}} .
\end{gathered}
$$

Здесь введены модуль Юнга $E_{0}$ жестко фиксированной консоли, максимальная деформация $D^{\max }=z(l)$, фактор коррекции $\Phi_{\mathrm{I}}, I=\pi d^{4} / 64$ для цилиндрической балки с диаметром $d$.

Схема модели I эквивалентна консоли на кольцевой пружине (рис. $1, b$, в центре). Момент сил $E I z^{\text {II }}$ [4], созданный пружиной, и угол отклонения консоли $z^{\mathrm{I}}$ линейно связаны. Изгиб такой консоли определяется решением уравнения $z^{\mathrm{IV}}=0$ с граничными условиями $z(0)=0$, $z^{\mathrm{II}}(0)=4 z^{\mathrm{I}}(0) / L, z^{\mathrm{II}}(X)=0, z^{\mathrm{III}}(X)=-F / E I$. Несложно убедиться (см. также дополнительные материалы в онлайн-версии статьи), что решением будет формула (1).

Внизу на рис. 1, $b$ показана схема балки на упругом основании с выносной консолью (модель II): первый пролет на упругом основании $x \in[-L, 0]$, коэффициент постели $k_{W}$; второй пролет $(x \in[0, l])$ подвешен. В общем виде изгиб первого пролета [9] задается линейной комбинацией $z_{1}(x)$ функций Крылова $Y_{i}$ (см. таблицу), а изгиб консоли - полиномом $z_{2}(x)$ :

$$
\begin{gathered}
z_{1}(x)=\sum_{i=1}^{4} A_{i} Y_{i}(\beta x), \beta=\sqrt[4]{\frac{k_{W}}{4 E I}}=\sqrt[4]{\frac{16 k_{W}}{\pi E}} d^{-1}, \\
z_{2}(x)=\sum_{i=0}^{3} a_{i} x^{i} .
\end{gathered}
$$

Для $I$ в (4) использован момент инерции сечения цилиндрической балки с диаметром $d$.

Был определен (см. дополнительные материалы в онлайн-версии статьи) аналитический вид $z_{1}(x)$ и $z_{2}(x)$ для граничных условий $z_{1}^{\mathrm{I}}(-L)=z_{1}(-L)=0, z_{1}^{\mathrm{II}}(0)=$ $=z_{2}^{\mathrm{II}}(0), z_{1}^{\mathrm{I}}(0)=z_{2}^{\mathrm{I}}(0)$ и $z_{1}(0)=z_{2}(0), z_{2}^{\mathrm{III}}(X)=-F / E I$ и $z_{2}^{\mathrm{II}}(X)=0$.
Основные соотношения модели II: формула подгонки к эксперименту

$$
\begin{gathered}
\xi_{\mathrm{II}}(\chi)=\left[3 K_{4}\left(2 \beta_{l} \Lambda\right)+6 K_{3}\left(2 \beta_{l} \Lambda\right)\left(\beta_{l} \chi\right)+6 K_{2}\left(2 \beta_{l} \Lambda\right)\left(\beta_{l} \chi\right)^{2}\right. \\
\left.+2\left(K_{1}\left(2 \beta_{l} \Lambda\right)-1\right)\left(\beta_{l} \chi\right)^{3}\right]\left[3 K_{4}\left(2 \beta_{l} \Lambda\right)+6 K_{3}\left(2 \beta_{l} \Lambda\right) \beta_{l}\right. \\
\left.+6 K_{2}\left(2 \beta_{l} \Lambda\right) \beta_{l}^{2}+2\left(K_{1}\left(2 \beta_{l} \Lambda\right)-1\right) \beta_{l}^{3}\right]^{-1} \\
\Lambda=L / l, \quad \beta_{l}=\beta l
\end{gathered}
$$

фактор коррекции

$$
\begin{gathered}
\Phi_{\mathrm{II}}=1+\frac{3 K_{4}\left(2 \beta_{l} \Lambda\right)+6 K_{3}\left(2 \beta_{l} \Lambda\right) \beta_{l}+6 K_{2}\left(2 \beta_{l} \Lambda\right) \beta_{l}^{2}}{2\left(K_{1}\left(2 \beta_{l} \Lambda\right)-1\right) \beta_{l}^{3}}, \\
E=E_{0} \Phi_{\mathrm{II}} .
\end{gathered}
$$

Функции Крылова $K_{i}$ приведены в таблице.

В моделях I и II по одному параметру подгонки: $\lambda$ (см. (3a)) и $\beta_{l}$ (см. (5a)); параметр $\Lambda$ в каждом испы-

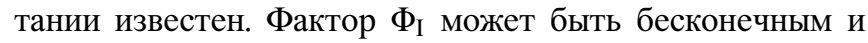
не зависит от жесткости подложки. Фактор $\Phi_{\text {II }}$ большой на мягких $\left(\beta_{l}\right.$ мал) и $\sim 1$ на твердых подложках. Можно ожидать, что модель I будет завышать значения модуля Юнга по сравнению с моделью II.

АСМ-методика испытаний подвешенного объекта на изгиб (модели I и II) были применены для определения модуля Юнга наносвитков состава $\mathrm{MgNi}_{2} \mathrm{Si}_{2} \mathrm{O}_{5}(\mathrm{OH})_{4}$. Из наносвитков, полученных методом гидротермального синтеза [10], была приготовлена суспензия в изопропаноле, капля которой наносилась и высыхала на кремниевой тестовой решетке TGZ2 (НT-МДТ СИ, Россия). Образцы исследовались в режиме ACM PeakForce QNM прибора BioScope Catalyst (Bruker, CША). Кроме деформации детектировались сигналы топографии и ошибки пиковой силы, которые нужны для корректировки значений деформации [6] с учетом вклада от проскальзывания АСМзонда на наклонных участках образца [11]. АСМ-данные обрабатывались в программе Gwyddion 2.55.

Из изображения скорректированной деформации извлекались два профиля вдоль наносвитка [5]. Длиной первого профиля считалась подвешенная часть наносвитка на изображении топографии, длина второго определялась по области ненулевой деформации. Нормированные по вертикали и горизонтали профили анализировались по моделям I и II для консолей и по алгоритму [5] для мостиков с подгоночной зависимостью

$$
\begin{aligned}
\xi(\chi)= & 4^{3}\left(\chi-\chi^{2}\right)^{3} \frac{2+\lambda}{(1+2 \lambda)(2+3 \lambda)} \\
& +4^{2}\left(\chi-\chi^{2}\right)^{2} \frac{6 \lambda(1+\lambda)}{(1+2 \lambda)(2+3 \lambda)}
\end{aligned}
$$

выражениями для фактора коррекции $Ф$ и модуля Юнга $E$

$$
\Phi=\frac{4 \lambda+2}{\lambda+2}, E_{C B}=\frac{F}{D^{\max }} \frac{l^{3}}{3 \pi d^{4}}, E=E_{C B} \Phi,
$$




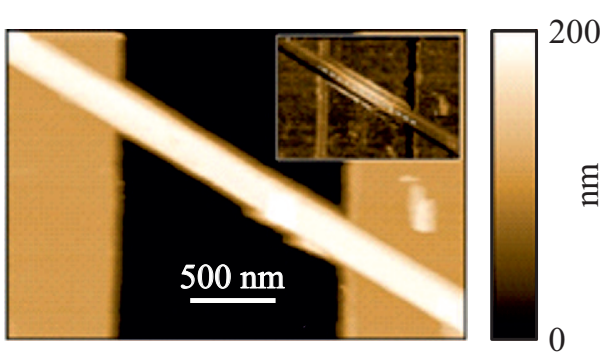

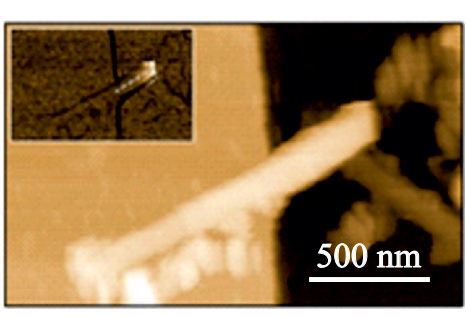
200

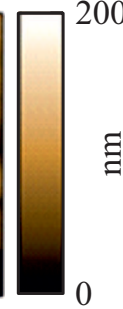

$\Xi$

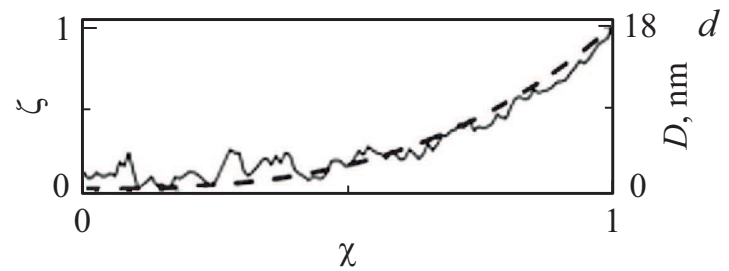

Рис. 2. АСМ-данные по высоте рельефа на участках TGZ2 с наносвитками $\mathrm{MgNi}_{2} \mathrm{Si}_{2} \mathrm{O}_{5}(\mathrm{OH})_{4}$, образующими мостик $(a)$ и консоль $(b)$ (на вставках показаны соответствующие данные для сигнала скорректированной деформации $D$; в обоих случаях перепад сигнала $0-20 \mathrm{~nm}$, профили $D$ извлекались вдоль пунктирной линии), и нормированные профили $\xi(\chi)$ мостика $(c)$ и консоли $(d)$. Параметры визуализации: жесткость кантилеверов FMG01 равна $2.4(a, c)$ и $3.6 \mathrm{~N} / \mathrm{m}(b, d)$; частота и амплитуда вертикальных осцилляций зонда $-1 \mathrm{kHz}$ и $150 \mathrm{~nm}$; частота строчной развертки - $0.3 \mathrm{~Hz}$; пиковая сила $F=80(a, c)$ и $15 \mathrm{nN}(b, d)$. На части $a$ длина пролета мостика $l=1701 \mathrm{~nm}$; на части $b$ длина консоли $l=417 \mathrm{~nm}$, а длина неподвешенной части балки $L=859 \mathrm{~nm}$.

$E_{C B}-$ модуль Юнга в случае защемленной балки, $\chi=X / l$ и $\lambda=L / l$ (см. рис. $1, a)$.

Из двух профилей деформации оставляли профиль с лучшей подгонкой (меньшей невязкой) кривыми (3a), (5a) или (6a). За внешний диаметр наносвитка $d$ была взята средняя высота неподвешенной части. Подставляя параметр подгонки, размеры подвешенной части, измеренное значение $F / D^{\max }$ в $(3 \mathrm{~b}),(5 \mathrm{~b})$ либо в (6b), получали искомую величину $E$.

На рис. 2 приведен пример АСМ-данных для мостика и консоли. Модуль Юнга мостика составил $E=134 \mathrm{GPa}$ при параметре подгонки $\lambda=0.42$, факторе коррекции $\Phi=1.52 \quad(l=1701 \mathrm{~nm}, d=81 \mathrm{~nm}$, $\left.F / D^{\max }=7.2 \mathrm{~N} / \mathrm{m}\right)$. Результаты анализа данных консоли по модели II: $E=63 \mathrm{GPa}, \beta_{l}=5.47, \Phi_{\mathrm{II}}=1.66$ $\left(l=417 \mathrm{~nm}, d=57 \mathrm{~nm}, \Lambda=2.06, F / D^{\max }=0.81 \mathrm{~N} / \mathrm{m}\right)$. Результаты для нее же по модели I: $E=63 \mathrm{GPa}$, $\lambda=0.87, \Phi_{\mathrm{I}}=1.65$. Такое совпадение модулей Юнга было случайным.

Большим $\beta_{l}$ в модели II и малым $\lambda$ в модели I соответствуют изгибы консоли по закону $\chi^{3}$. Чаще наблюдались сильные отклонения от $\chi^{3}$. Из 18 консолей, согласно модели I, пять изгибались по закону $\chi^{2}$, имея бесконечное $\Phi_{\mathrm{I}}$. Средние значение по оставшимся 13 консолям $E=496 \pm 1057 \mathrm{GPa}\left(\Phi_{\mathrm{I}}=7.42 \pm 13.30\right)$. Для 12 исследованных мостиков $E=134 \pm 148 \mathrm{GPa}$ $(\Phi=1.52 \pm 0.56)$, что меньше в $\sim 4$ раза. Напротив, хорошее согласие с модулем Юнга мостиков дал анализ всех 18 консолей по модели II: $E=109 \pm 86 \mathrm{GPa}$ $\left(\Phi_{\mathrm{II}}=3.05 \pm 1.15\right)$. Поэтому в испытаниях консолей предпочтителен анализ по модели II.
Рассмотрим $k_{W}$ - связь погонной силы и смещения упругого основания в модели II. По 18 испытаниям среднее значение $\left(\beta_{l} d / l\right)^{4}=0.072$. Согласно (4), $\left(\beta_{l} d / l\right)^{4}=16 k_{W} / \pi E$ и $k_{W}=0.014 E$. Жесткий цилиндр, наносвиток длиной $L$, вдавливается на глубину $z_{i}$ в мягкую подложку силой $F_{i}: F_{i} / L \approx\left[\pi E_{S} / 4\left(1-v_{S}^{2}\right)\right] z_{i}[12], E_{S}$ и $v_{S}$ - модуль Юнга и коэффициент Пуассона подложки. Отсюда $E_{S} \approx k_{W}=0.014 E \approx 2 \mathrm{GPa}(E-$ модуль Юнга наносвитка). У выступов решетки $\mathrm{SiO}_{2} \mathrm{TGZ2}$ модуль Юнга $70 \mathrm{GPa}$, поэтому значение $E_{S}$, по-видимому, характеризует загрязнения решетки и наносвитков.

В заключение отметим, что предложена улучшенная АCМ-методика испытаний подвешенного объекта на изгиб, учитывающая как он закреплен на подложке. Изучены две модели условий закрепления консолей. Согласованный с модулем Юнга мостиков результат достигается только для модели балки на упругом основании с выносной консолью.

\section{Благодарности}

Авторы благодарят А.А. Красилина за предоставление и подготовку к АСМ-исследованиям образцов синтетических наносвитков состава $\mathrm{MgNi}_{2} \mathrm{Si}_{2} \mathrm{O}_{5}(\mathrm{OH})_{4}$ и М.Б. Бабенкова за помощь в расчетах.

\section{Финансирование работы}

Работа выполнена при поддержке Российского научного фонда (грант № 19-13-00151). 


\section{Конфликт интересов}

Авторы заявляют, что у них нет конфликта интересов.

\section{Список литературы}

[1] J.-P. Salvetat, A.J. Kulik, J.-M. Bonard, G.A.D. Briggs, T. Stöckli, K. Méténier, S. Bonnamy, F. Béguin, N.A. Burnham, L. Forró, Adv. Mater., 11 (2), 161 (1999). DOI: $\quad 10.1002 /($ SICI $) 1521-4095(199902) 11: 2<161:: A I D-$ ADMA161 >3.0.CO;2-J

[2] S. Cuenot, S. Demoustuer-Champagne, B. Nysten, Phys. Rev. Lett., 85 (8), 1690 (2000). DOI: $10.1103 /$ PhysRevLett.85.1690

[3] A. Kis, Mechanical properties of mesoscopic objects, $\mathrm{PhD}$ thesis (EPFL, Lausanne, 2003).

[4] L.D. Landau, E.M. Lifshitz, Theory of elasticity (Pergamon, Oxford, 1970), p. 89.

[5] A.V. Ankudinov, Semiconductors, 53(14), 1891 (2019). DOI: $10.1134 / \mathrm{S} 1063782619140021$

[6] M.M. Khalisov, V.A. Lebedev, A.S. Poluboyarinov, A.V. Garshev, E.K. Khrapova, A.A. Krasilin, A.V. Ankudinov, Nanosyst: Phys., Chem., Math., 12 (1), 118 (2021).

DOI: $10.17586 / 2220-8054-2021-12-1-118-127$

[7] D. Gangadean, D.N. McIlroy, B.E. Faulkner, D.E. Asto, Nanotechnology, 21, 225704 (2010).

DOI: $10.1088 / 0957-4484 / 21 / 22 / 225704$

[8] С.П. Тимошенко, Сопротивление материалов: элементарная теория и задачи (Наука, М., 1965), т. 1, с. 155.

[9] А.Н. Крылов, О расчете балок, лежсащих на упругом основании (АН СССР, Л., 1931), с. 24.

[10] E.K. Khrapova, V.L. Ugolkov, E.A. Straumal, S.A. Lermontov, V.A. Lebedev, D.A. Kozlov, T.S. Kunkel, A. Nominé, S. Bruyere, J. Ghanbaja, T. Belmonte, A.A. Krasilin, ChemNanoMat, 7 (3), 257 (2021).

DOI: $10.1002 / \mathrm{cnma} .202100018$

[11] А.В. Анкудинов, М.М. Халисов, ЖТФ, 90 (11), 1951 (2020). DOI: 10.21883/JTF.2020.11.49989.117-20 [A.V. Ankudinov, M.M. Khalisov, Tech. Phys., 65 (11), 1866 (2020). DOI: 10.1134/S1063784220110031].

[12] V.L. Popov, Contact mechanics and friction: physical principles and applications (Springer, Germany, 2017), p. 57. DOI: 10.1007/978-3-662-53081-8_18 